\title{
James Webb Space Telescope Integrated Science Instrument Module Thermal Balance/Thermal Vacuum Test Configuration and Test Planning at NASA's Goddard Space Flight Center
}

\author{
Stuart Glazer ${ }^{1}$ and Paul Cleveland ${ }^{2}$ \\ ${ }^{I}$ NASA/Goddard Space Flight Center, Greenbelt, Maryland, USA, 20771 \\ ${ }^{2}$ Energy Solutions International LLC, Laytonsville, Maryland, USA, 20882
}

\begin{abstract}
The James Webb Space Telescope (JWST) is the next of the "great observatories", scheduled to be launched in 2014. Three of the four science instruments are passively cooled to their operational temperature range of $36 \mathrm{~K}$ to $40 \mathrm{~K}$, and the fourth instrument is actively cooled to its operational temperature of approximately $6 \mathrm{~K}$. Thermal-vacuum testing of the flight science instruments at the Integrated Science Instrument Module (ISIM) element level will take place within a newly constructed shroud cooled by gaseous helium inside Goddard Space Flight Center's (GSFC) Space Environment Simulator (SES). The test enclosure surrounding the instruments during the integrated ISIM-level thermal balance testing is complex, and is designed to simulate as closely as possible the in-flight conductive and radiative thermal environment around the ISIM. Thermal control and measurement of parasitic sources of heat leak into the test volume is critical, as the dissipation plus known parasitics in the flight ISIM is approximately $454 \mathrm{~mW}$, and additional parasitics attributed to the flight enclosure itself are nearly equal to this, resulting in the energy balance of the inflight ISIM being less than 1.0 W. Sources of test-induced parasitics must be carefully controlled and measured, and the ability to thermally control the test environment is critical to enable accurate thermal balance testing and thermal model correlation. This paper describes the test configuration and plans for the ISIM-level thermal vacuum/thermal balance testing at GSFC.
\end{abstract}

\section{Introduction}

$\mathrm{T}$ he James Webb Space Telescope (JWST) is the next of the "great observatories", designed to be launched into orbit around the second Lagrange point in 2014. It has a $6.5 \mathrm{~m}$ (deployed) multi-faceted mirror, and its science instruments operate in short to medium wavelengths, to enable insights to the formation of the galaxies and planets following the "Big Bang". NASA's Goddard Space Flight Center (GSFC) is the mission lead, and is directly responsible for the Integrated Science Instrument Module (ISIM), including its integration and test. The suite of four science instruments, located in the cryogenic volume behind the primary reflector (denoted as Region 1), is comprised of the Near Infrared Spectrograph (NIRSpec), primarily sponsored by the European Space Agency (ESA) with substantial NASA contribution; the actively cooled Mid Infrared Instrument (MIRI), jointly sponsored by ESA and the European Consortium (EC) with support from the Jet Propulsion Laboratory (JPL); the Fine Guidance Sensor (FGS), provided by the Canadian Space Agency (CSA); and the Near Infrared Camera (NIRCam), provided by NASA. In flight, NIRSpec, NIRCam, and FGS are cooled to the range of 36 to $40 \mathrm{~K}$ passively via heat transfer through complex high-purity aluminum thermal links to dedicated radiators, while the MIRI is actively cooled to approximately $6 \mathrm{~K}$. NASA provides the cooler and supplemental hardware for MIRI. The cooler's compressor is

\footnotetext{
${ }^{1}$ James Webb Space Telescope ISIM Thermal Systems Lead, Thermal Engineering Branch, Code 545.3

${ }^{2}$ James Webb Space Telescope ISIM Thermal Test Engineer
} 
located in the ambient temperature spacecraft bus, outside the cryogenic Region 1 . The science instruments are mounted on the ISIM structure, a special composite truss structure designed for exceptional optical stability, provided by NASA. The Observatory Prime Contractor is Northrop Grumman Aerospace Systems (NGAS).

Figures I-1 and I-2 illustrate the observatory and the major components of the ISIM, respectively. The ISIM Electronics Compartment (IEC), in close proximity to Region 1, houses the instrument and detector control electronics, and operates at approximately ambient temperature. It is denoted as "Region 2". The ISIM Harness Radiator (IHR), which operates below $80 \mathrm{~K}$ and is also provided by ISIM, rejects most harness parasitic heat loads to space before the harnesses enter Region 1. The ISIM Thermal Control Subsystem provides 93 flight housekeeping temperature sensors on the instruments, ISIM structure, radiators, and other locations, and trim heaters to guarantee minimum instrument operational temperatures. It arranges for control of contamination control heaters on each instrument (except the MIRI), to avoid contamination during post-launch cooldown, and in the event of contamination on-station.

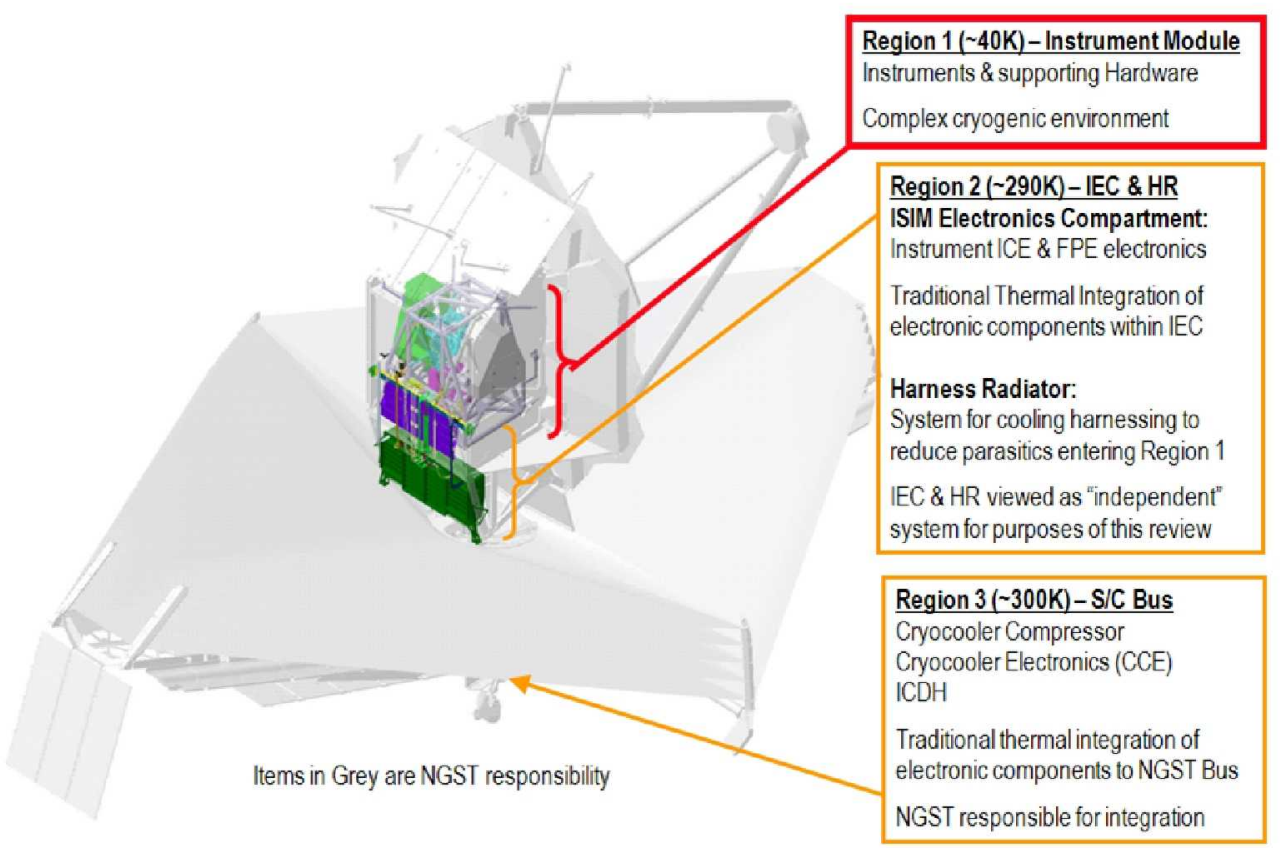

Figure I-1. JWST Observatory

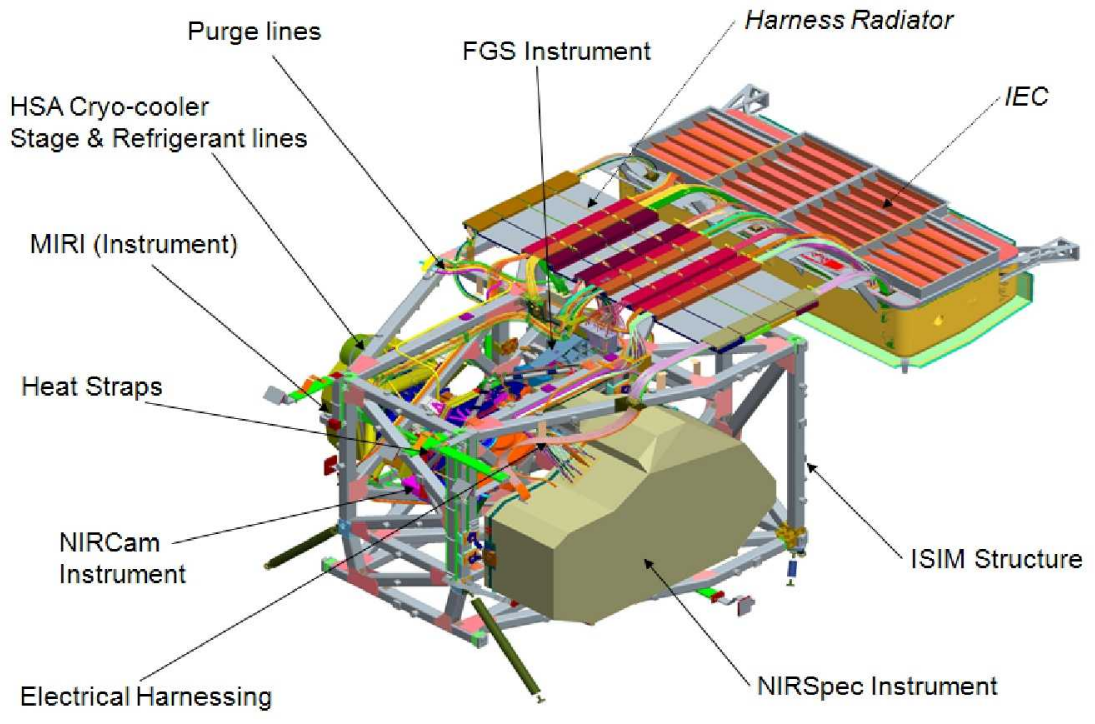

Figure I-2. Integrated Science Instrument Module, with Harness Radiator and Integrated Electronics Compartment 
Previous papers ${ }^{1,2}$ have described the general characteristics of the James Webb Space Telescope (JWST). Since those papers were written, some important changes have taken place in the observatory thermal design and the test program. First, the method of cooling the MIRI science instrument to approximately $6 \mathrm{~K}$ has changed from solid hydrogen stored within a dewar to a closed cycle mechanical cooler. While this potentially lengthens the observing time for the instrument, the cooler has a limited design heat lift capability, and the total heat load to the first stage must fall within that capability in order for the instrument to reach its operational temperature. Initial thermal balance tests of the Verification Model of the MIRI indicated slightly higher radiative heat loads than originally predicted through its multi-layer insulation (MLI), when exposed to the worst case hot $41 \mathrm{~K}$ thermal environment inside the ISIM. To recover lost margin to the cooler's heat lift capability, the MLI covering the instrument was redesigned by the instrument team, and a decision was made by NASA (responsible for the ISIM) to provide a radiative "shield" around the MIRI within the ISIM enclosure. The shield is to be cooled to approximately $18 \mathrm{~K}$ by means of a conductive link to the cooler's second stage. Detail design of the shield is ongoing at this time.

\section{Overview of Facilities Used for ISIM-level Thermal Vacuum Testing}

The thermal test program of the ISIM has evolved substantially since 2007; most notably, the decision was made to forego thermal vacuum/thermal balance testing of the Engineering Test Units (ETU) of the instruments at the integrated ISIM level. This resulted in the decision to produce only a single protoflight ISIM structure, rather than a prototype and flight unit. Current plans still call for pre- and post-vibration thermal vacuum/thermal balance testing, combined with optical, electrical, and other performance testing at the Goddard Space Flight Center for the flight units, prior to integration with the Optical Telescope Element (OTE) and subsequent thermal vacuum testing at the Johnson Space Center (JSC). The thermal tests at the ETU level were originally intended for risk reduction, providing performance confirmation at an early stage. They were also to be used as a "dry-run" or pathfinder, for the flight unit tests at the ISIM-level, verifying that: 1) test Ground Support Equipment (GSE) and test procedures were designed correctly and performed adequately to yield desired test conditions; 2) test profiles were optimized and facility capabilities were well understood and matched to the test needs; 3 ) test-induced parasitic heat loads were characterized and quantified, and would not interfere with model correlation; and 4) instrumentation measurement accuracy would be adequate for thermal model validation. It was desired to obtain this information prior to the flight unit testing because of the high cost of the test in the Space Environment Simulator (SES), NASA GSFC's largest thermal vacuum chamber, and the projected test durations of nearly five months per test. Further, because the total heat load to the flight units is expected to be less than 1.0 watt, it is likely that small unknown sources of parasitic heat would not manifest themselves until flight unit temperatures approached operational balance conditions, occurring approximately 30 days after the test start.

A new removable and reconfigurable Gaseous Helium (GHe) shroud was recently designed and fabricated to support JWST testing at GSFC. It was designed with a diameter of $7.62 \mathrm{~m}(25.5 \mathrm{ft})$, and fits within the fixed $\mathrm{LN}_{2}$ shroud which has a diameter of $8.23 \mathrm{~m}(27.0 \mathrm{ft})$ inside the SES. Figures II-1 and II-2 show the GHe shroud in two different height configurations which will be used for: 1) ISIM-level thermal vacuum/thermal balance (TV/TB) testing; and 2) Optical Telescope Element (OTE) hardware thermal testing, respectively (the non-ISIM OTE test article is larger than the ISIM). In the configuration supporting the ISIM-level testing, the GHe shroud has an enclosed height of $4.57 \mathrm{~m}(15.0 \mathrm{ft})$, while in the OTE test configuration its enclosed height is $8.37 \mathrm{~m}(27.5 \mathrm{ft})$. A more complete description of the new helium shroud, including performance capabilities obtained from initial tests, is given in a companion paper ${ }^{3}$. As shown in Figure IV-3, the flight ISIM and associated GSE will be completely enclosed within the upper volume during ISIM-level TV/TB testing, which is cooled with GHe to approximately 18K. The Optical Simulator (OSIM), a critical piece of optical GSE used to evaluate instrument optical performance, is positioned beneath the cooled GHe shroud floor, and provides a beam into the instruments pick-off mirrors from below. The OSIM must be kept at $100 \mathrm{~K}$, so it is surrounded by the facility's fixed $\mathrm{LN}_{2}$ shroud, partially blanketed, and controlled to a stable temperature with heaters. Similarly, the large Vibration Isolation System (VIS), upon which the entire flight ISIM is supported from the chamber floor, must operate at ambient temperature. It too is beneath the helium shroud, and is heavily blanketed and heated to maintain it at ambient temperature. Its heat load ultimately goes to the chamber Nitrogen shroud system. 


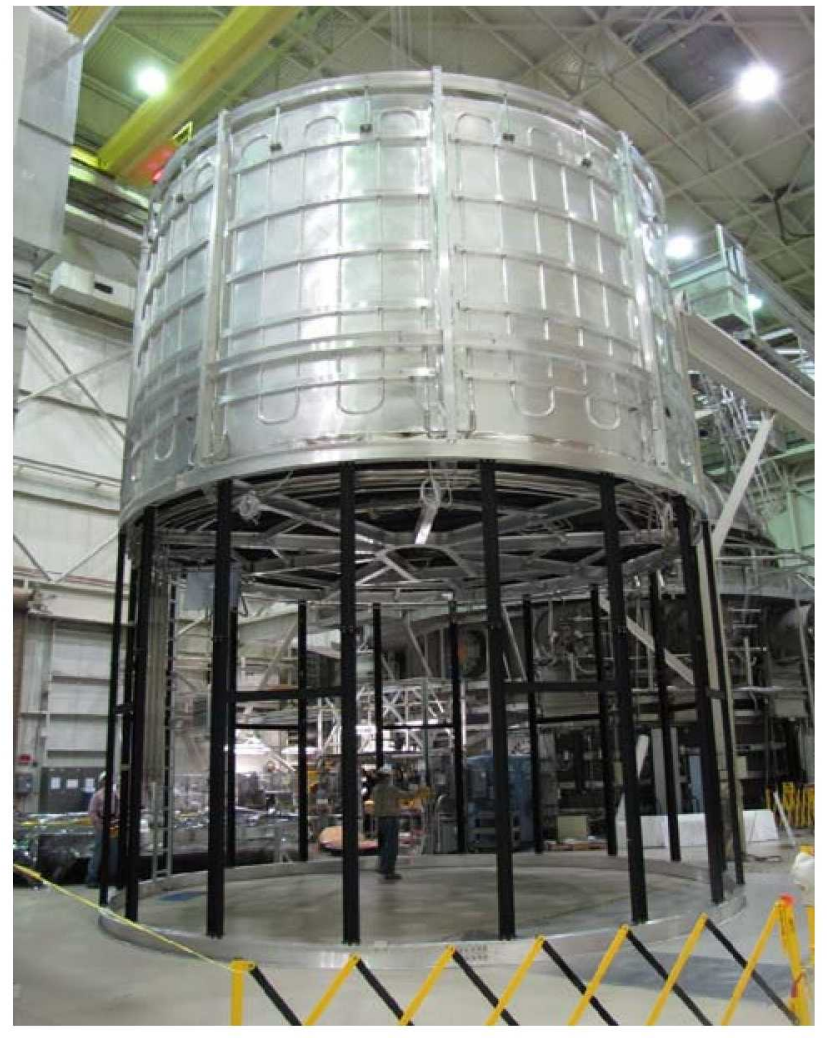

Figure II-1. SES GHe Shroud in -01 (ISIM) Test Configuration

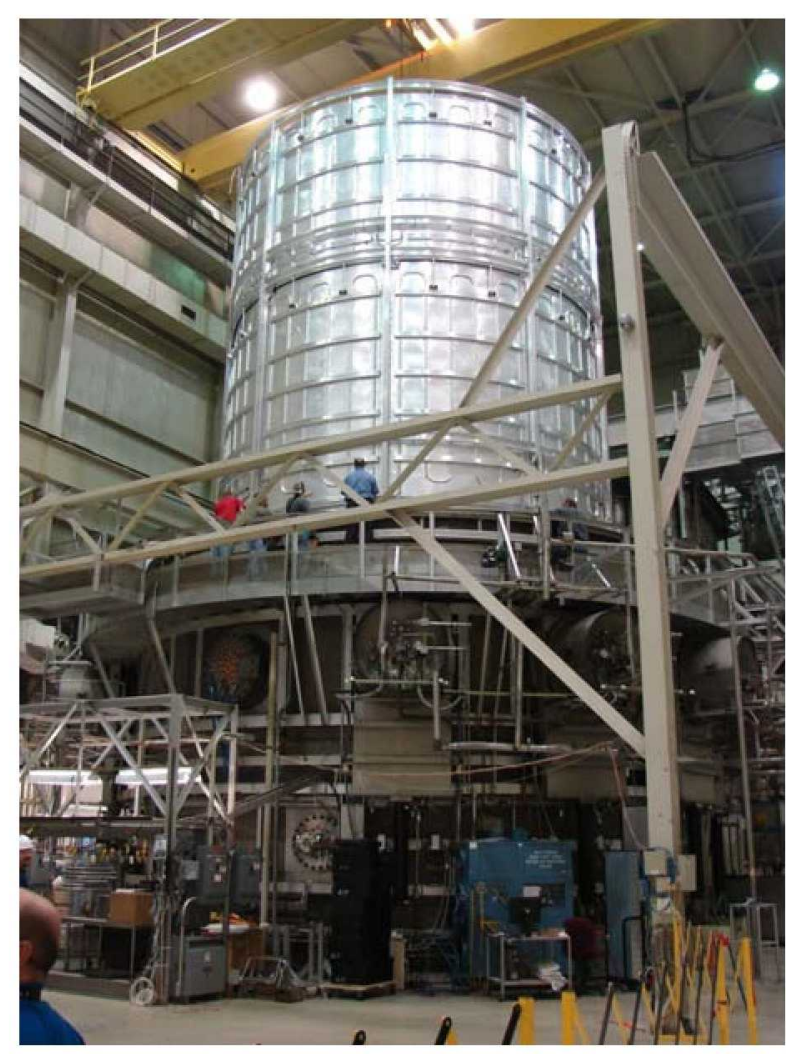

Figure II-2. SES GHe Shroud in -03 (OTE GSE) Test Configuration

The JWST and ISIM management have defined a series of precursor thermal tests in the SES facility, designed to incrementally check nearly all aspects of the facility and GSE performance prior to thermal vacuum testing of the flight ISIM. Figure II-3 is a timeline of the major ISIM tests currently scheduled in the SES. The first facility test was the He Shroud Configuration -03 Acceptance Test in June 2009. This reflected the larger He shroud configuration which will support the OTE CryoSet test. The second facility test was the SES Chamber Hardware Certification Test in early 2010 which reflected the smaller -01 configuration. Test results were excellent, confirming adequate He refrigerator heat lift capacity, reliability, shroud temperature stability and gradient control, and vacuum retention. Those tests have also helped evaluate: planned test procedures; adequacy of the thermal control hardware affixed to critical GSE; operational performance characterization of the specialized photogrammetry equipment and its thermal control system; and dimensional alignment stability of the critical ISIM Test Platform (ITP), used to support the flight ISIM in test. Additional follow-on tests include:

1. Cryo-Set test of the flight ISIM structure, in which the structure is thermally cycled several times from ambient to cryogenic temperature, while its optical alignment and stability are measured in-situ with the specialized photogrammetry equipment;

2. Cryo-Proof test of the flight ISIM structure, which verifies its structural integrity and alignment stability using mass simulators in place of the flight instruments;

3. OSIM CryoCal test of the OSIM optical GSE, which verifies its performance at cryogenic conditions;

4. OTE Cryo-Set test, which exposes the flight OTE backplane structure to thermal vacuum cycling from ambient to cryogenic temperatures;

5. Cryo-Vac Test \#1, the pre-vibration thermal vacuum and thermal balance testing of the flight ISIM;

6. Cryo-Vac Test \#2, the post-vibration thermal vacuum and thermal balance testing of the flight ISIM. 


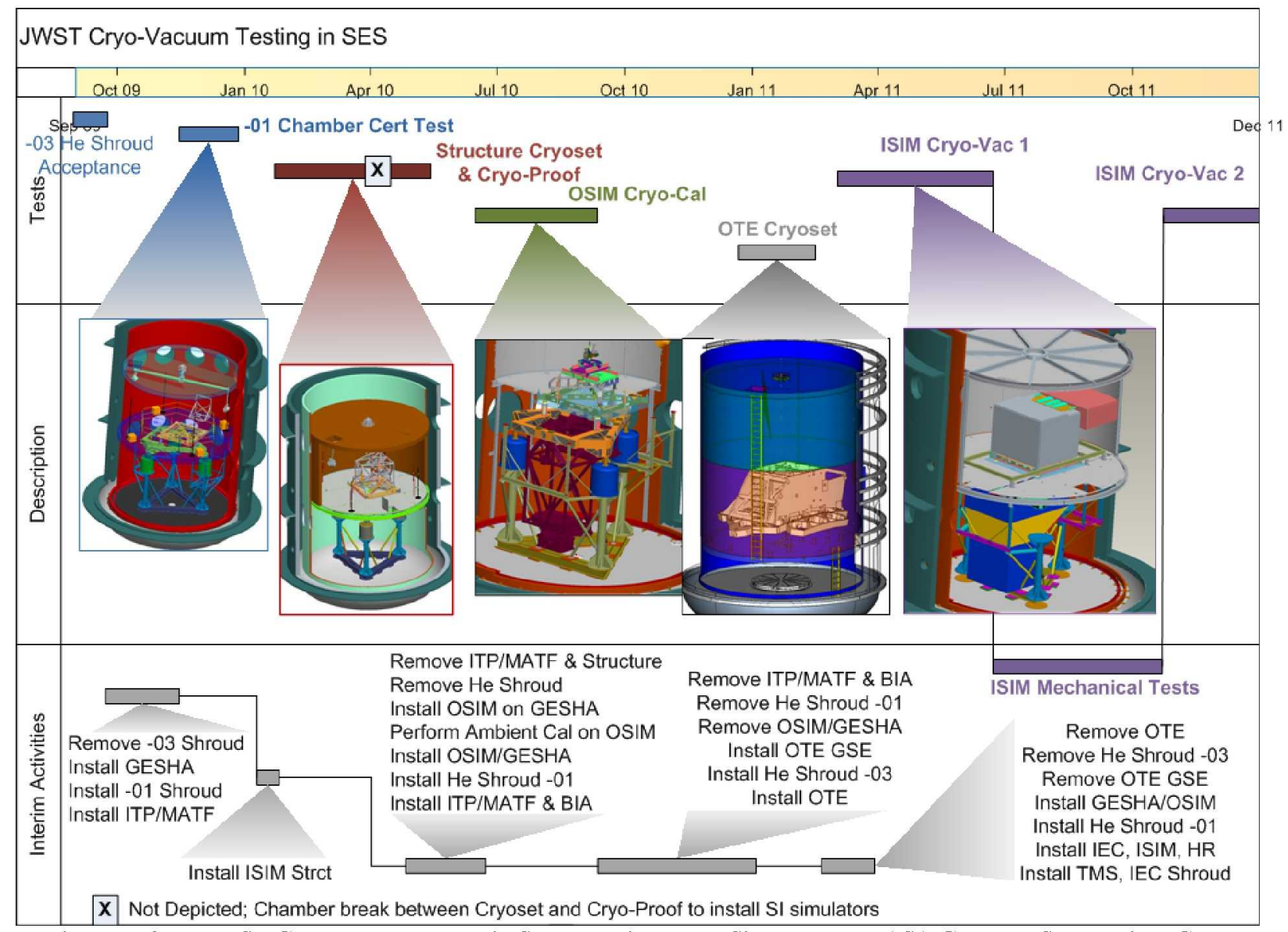

Figure II-3. JWST Cryo-Vacuum Tests in Space Environment Simulator at NASA Goddard Space Flight Center

\section{Flight ISIM-level Cryo-Vac Thermal Test Objectives}

The flight ISIM must be fully verified at the ISIM-level at GSFC before it is integrated to the OTE. As a result, the thermal test environment provided in the ISIM-level Cryo-Vac tests must be flight-like to permit realistic performance verification and characterization of the ISIM instruments, their optics, electronics, software, and all other subsystems. Detailed thermal requirements are defined for instrument temperature control, thermal stability, electrical harness parasitics, heat transfer to the instrument radiators, contamination avoidance during cooldown and decontamination (if needed) on-station, etc. The absence of the flight thermal environment to the ISIM, plus the presence of substantial GSE, makes it difficult to reproduce precisely the cryogenic flight environment in test. Thus, while there is a strong desire to verify by test as many thermal requirements levied on the ISIM Thermal Control Subsystem as possible, the final verification for many will take place by analysis using a thermal model correlated with test data.

Key ISIM-level Cryo-Vac test objectives leading to thermal system verification and characterization include:

- ISIM thermal model validation;

- Multi-Layer Insulation (MLI) performance and workmanship;

- End-to-end (instrument to radiator) heat strap thermal conductance at operational temperatures;

- Heat transfer to the instrument radiators;

- Harness parasitic radiator performance and parasitic harness heat loads to ISIM structure, each instrument, and to the Backplane Support Fixture (BSF) ( to which ISIM is mounted);

- Performance (adequacy), workmanship of housekeeping temperature sensors and operation of contamination control heaters/control algorithms during cooldown and warmup;

- Trim heater capability to meet minimum operating temperature requirements for the instruments in general, and their Application Specific Integrated Circuits (ASIC);

- Science instrument transient stability during multi-instrument operation;

- Sensitivity of ISIM to backplane interface temperature; 
- MIRI thermal performance, heat load measurement, within shield enclosure;

- IEC thermal balance, cycling, transient stability, steady state surface and box mounting temperatures

\section{Flight ISIM-level Cryo-Vac Test Configuration, Test Planning}

The ISIM element will provide a flight-like thermal environment to the Region 1 components during the CryoVac tests.

\section{A. Thermal Environment Simulation}

Close inspection of the ISIM components in Region 1 reveals that there are 18 conductive interfaces to the surroundings, illustrated in Figure IV-1. These interface points include kinematic mounts between the ISIM structure and the BSF, heat strap attachments to instrument radiators (and mechanical mounting to the BSF in a few locations), and a purge line interface. The complete radiative environment surrounding the flight ISIM in Region 1, made up primarily by the "ISIM Enclosure" (provided by the prime contractor), may be approximated with 10 discrete surfaces (the conical Aft Optics volume, present in the flight observatory, is not simulated in test). Figure IV-2 shows these 10 radiative interfaces to Region 1.
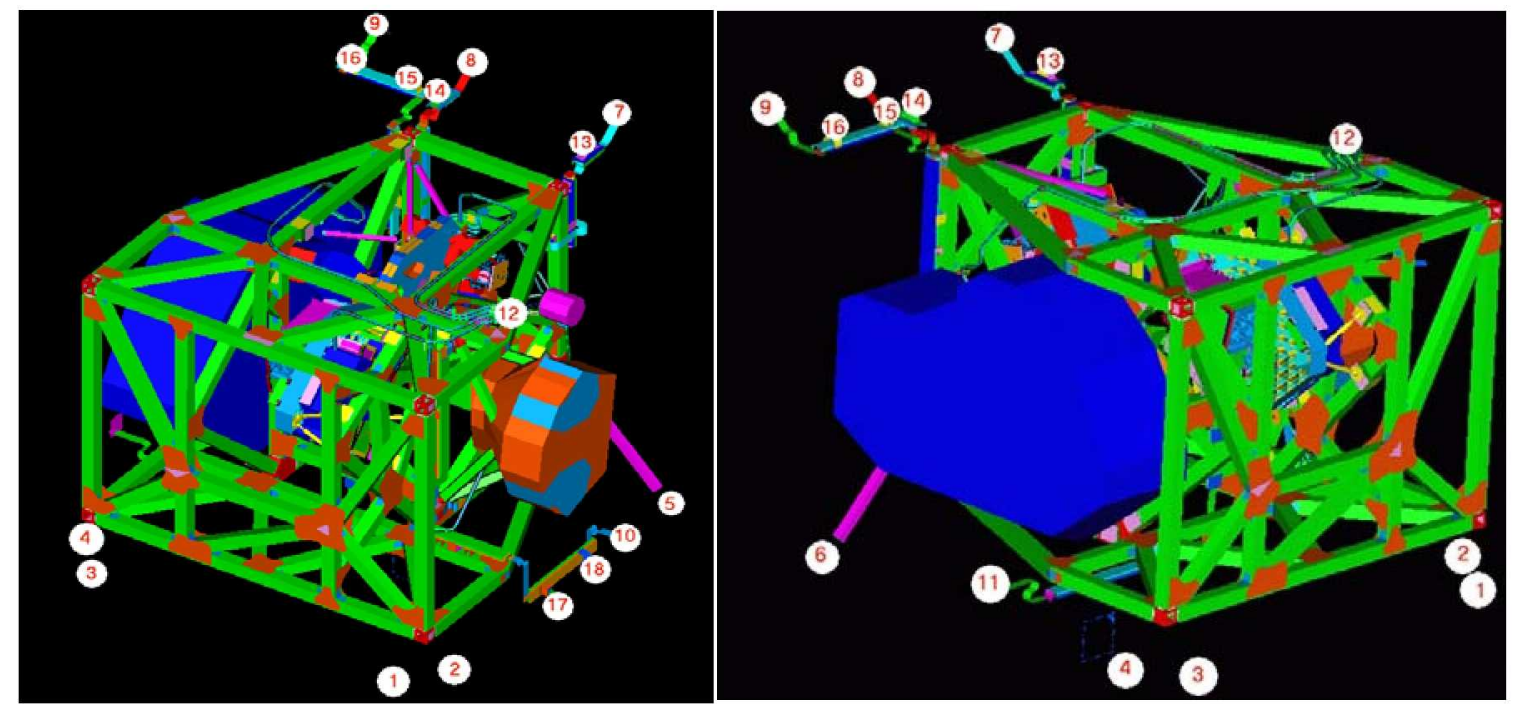

Figure IV-1. Conductive Interfaces to ISIM Region 1
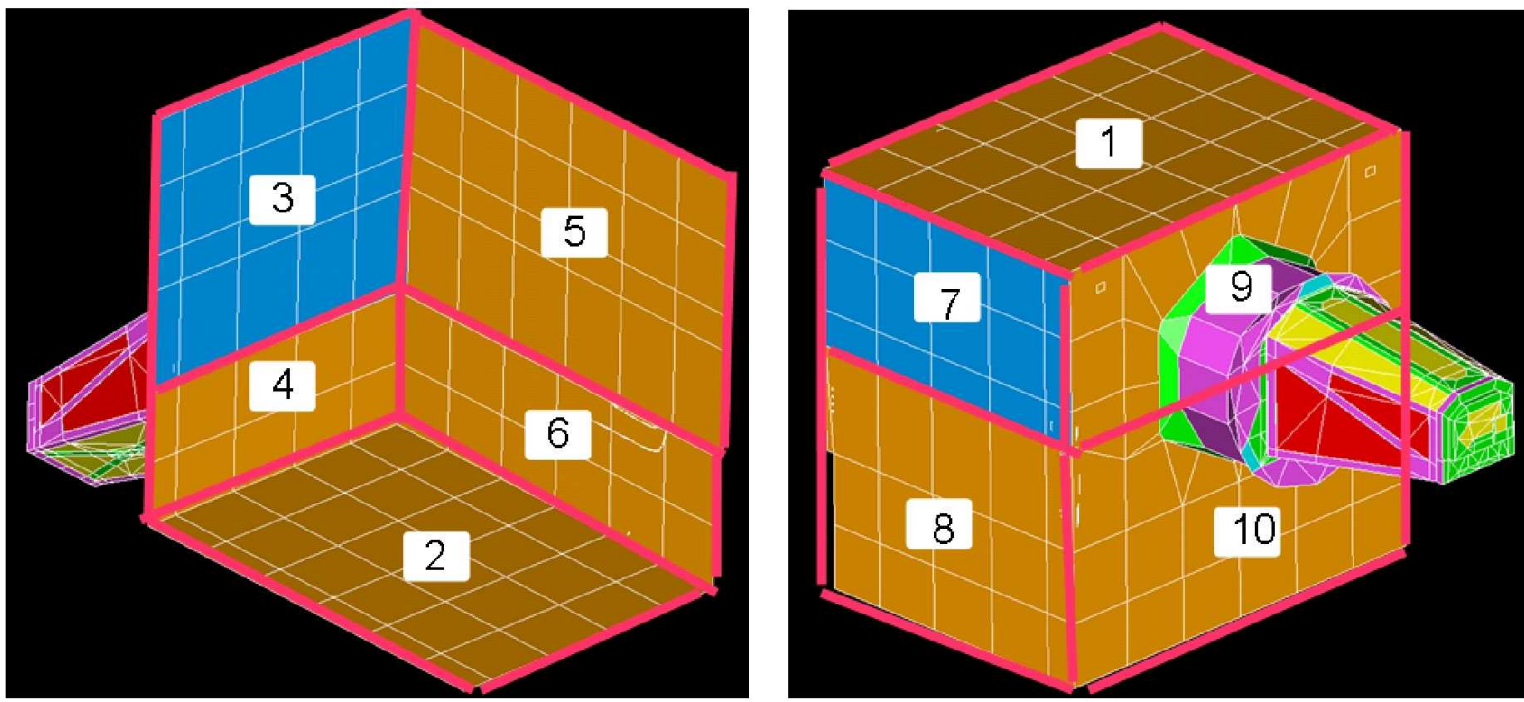

Figure IV -2. Radiative Interfaces Surrounding ISIM Region 1 (simulating ISIM Enclosure)

6

American Institute of Aeronautics and Astronautics 
Each of the 18 conductive interfaces to Region 1 identified above will be simulated in test with individually temperature controlled GSE, since neither the flight BSF nor the instrument radiators are present during the ISIMlevel Cryo-Vac tests. The ITP, a GSE invar structure designed to simulate the structural mounting characteristics of the flight BSF, replaces it during the test. The flight thermal links, or heat straps, that attach the NIRSpec instrument, NIRCam instrument, FGS instrument, NIRSpec detector and ASIC, and the MIRI support structure mount feet and harnesses to their respective radiators for thermal control, will be attached in test to individually controlled heat sinks through specially designed "Q-meters" (simple devices that measure heat flows). The BSF purge line will also be temperature controlled at the interface to the ISIM. Similarly, each of the 10 radiative panels provided in test are to be individually temperature controlled, with ISIM-facing surfaces covered with the same materials and/or coatings as the flight ISIM enclosure. The GSE conductive and radiative thermal interfaces provided in test, its support structures, plus all included thermal control and measurement instrumentation, constitute the Surrogate Thermal Management System (STMS). The STMS conductive and radiative interfaces are listed in Tables IV-1 and IV-2, respectively.

Table IV-1. Conductive Interfaces to ISIM Region 1

\begin{tabular}{|c|l|l|}
\hline I/F & \multicolumn{1}{|c|}{ Interface Location } & \multicolumn{1}{|c|}{ Controlled STMS Location } \\
\hline 1 & $-\mathrm{V} 2 /$-V3 KM - Bipod leg A (-V3) & At ITP KM mount point \\
\hline 2 & $-\mathrm{V} 2 /-\mathrm{V} 3 \mathrm{KM}-$ Bipod leg B (+V3) & At ITP KM mount point \\
\hline 3 & $+\mathrm{V} 2 /-\mathrm{V} 3 \mathrm{KM}-$ Bipod leg A (-V3) & At ITP KM mount point \\
\hline 4 & $+\mathrm{V} 2 /-\mathrm{V} 3 \mathrm{KM}-$ Bipod leg B (+V3) & At ITP KM mount point \\
\hline 5 & $-\mathrm{V} 2$ Monopod KM & At ITP KM mount point \\
\hline 6 & + V2 Monopod KM & At ITP KM mount point \\
\hline 7 & Heat Strap I/F to Radiator: NIRCam & At Q-meter I/F to heat strap \\
\hline 8 & Heat Strap I/F to Radiator: NIRSpec FPA/ASIC & At Q-meter I/F to heat strap \\
\hline 9 & Heat Strap I/F to Radiator: NIRSpec OA & At Q-meter I/F to heat strap \\
\hline 10 & Heat Strap I/F to Radiator: MIRI & At Q-meter I/F to heat strap \\
\hline 11 & Heat Strap I/F to Radiator: FGS & At Q-meter I/F to heat strap \\
\hline 12 & Purge line I/F to BSF & At external GSE I/F to purge line \\
\hline 13 & Heat Strap support I/F to BSF: NIRCam & At external GSE I/F to heat strap supports \\
\hline 14 & Heat Strap support I/F to BSF: NIRSpec FPA/ASIC & At external GSE I/F to heat strap supports \\
\hline 15 & Heat Strap support I/F to BSF: NIRSpec OA (1) & At external GSE I/F to heat strap supports \\
\hline 16 & Heat Strap support I/F to BSF: NIRSpec OA (2) & At external GSE I/F to heat strap supports \\
\hline 17 & Heat Strap support I/F to BSF: MIRI (1) & At external GSE I/F to heat strap supports \\
\hline 18 & Heat Strap support I/F to BSF: MIRI (2) & At external GSE I/F to heat strap supports \\
\hline
\end{tabular}


Table IV-2. Radiative Interfaces to ISIM Region 1

\begin{tabular}{|c|l|}
\hline I/F & \multicolumn{1}{|c|}{ Controlled STMS Surface } \\
\hline 1 & $+\mathrm{V} 3$ Enclosure Panel \\
\hline 2 & $-\mathrm{V} 3$ Enclosure Panel \\
\hline 3 & $+\mathrm{V} 2 /+\mathrm{V} 3$ Enclosure Panel \\
\hline 4 & $+\mathrm{V} 2 /-\mathrm{V} 3$ Enclosure Panel \\
\hline 5 & $-\mathrm{V} 1 /+\mathrm{V} 3$ Enclosure Panel \\
\hline 6 & $-\mathrm{V} 1 /-\mathrm{V} 3$ Enclosure Panel \\
\hline 7 & $+\mathrm{V} 2 /+\mathrm{V} 3$ Enclosure Panel \\
\hline 8 & $+\mathrm{V} 2 /-\mathrm{V} 3$ Enclosure Panel \\
\hline 9 & $+\mathrm{V} 1 /+\mathrm{V} 3$ Enclosure Panel \\
\hline 10 & $+\mathrm{V} 1 /$-V3 Enclosure Panel \\
\hline
\end{tabular}

The flight Instrument Electronics Compartment (Region 2) will be present in test, but it will be entirely enclosed within a double shroud. An inner shroud cooled with $\mathrm{LN}_{2}$ will accept most of the $230 \mathrm{~W}$ (allocated) of heat load from the electronics boxes.An outer GHe cooled shroud will maintain an exterior surface temperature consistent with the GHe chamber shroud. This prevents "pollution" of the GHe chamber shroud volume with short wavelength energy which might leak into the critically controlled Region 1 within the STMS. Similarly, the flight Instrument Harness Radiator, designed to minimize heat transfer to Region 1 from the harnesses as they transition from the ambient temperature Region 2 IEC, will be provided a separate temperature controlled environment.

Radiative panels of the STMS will be plumbed for transport of GHe from the facility refrigerator for rapid transition to cryogenic temperature at the start of the test, and for radiant environment control to prevent contamination at the test conclusion. Once the panels reach their approximate operational temperatures, the helium flow in each panel will be stopped, and fine temperature control will be provided by heat strap connections to coldplates plus controlled heat addition. Conductive interfaces to the ISIM structure are provided by individually temperature controlled ISIM kinematic mount surfaces on the ITP (the ITP itself incorporates GHe cooling lines and heater control). Temperature sensors and controlled heater circuits at each controlled conductive interface point and on each temperature controlled radiant panel will be fully redundant to ensure test continuity throughout the estimated 5-month duration of each Cryo-Vac test. Table IV-3 lists preliminary thermal control and measurement requirements for the STMS radiative surfaces and conductive interface points.

Table IV-3. Preliminary Thermal Control Requirements for STMS Panel and Interface Points

\begin{tabular}{|c|c|c|c|c|c|c|c|}
\hline \multirow{2}{*}{\begin{tabular}{l}
\multicolumn{1}{c}{ Parameter } \\
Controlled I/F and Panel \\
Temperature range $(\mathbf{K})$
\end{tabular}} & \multirow{2}{*}{\begin{tabular}{c|}
$\begin{array}{c}\text { Cryo: } \\
\text { Balance }\end{array}$ \\
30 to 50 \\
\end{tabular}} & \multirow{2}{*}{$\begin{array}{c}\begin{array}{c}\text { Cryo: Non- } \\
\text { Balance }\end{array} \\
30 \text { to } 50 \\
\end{array}$} & \multicolumn{5}{|c|}{$\begin{array}{l}\text { Transition from Ambient to Bakeout, or from } \\
\text { Bakeout to Cryo, or from Cryo to Ambient }\end{array}$} \\
\hline & & & 18 to 50 & $\begin{array}{c}50 \text { to } \\
100\end{array}$ & $\begin{array}{c}100 \text { to } \\
200\end{array}$ & $\begin{array}{c}200 \text { to } \\
313\end{array}$ & $\begin{array}{c}313 \text { to } \\
370\end{array}$ \\
\hline $\begin{array}{l}\text { I/F Point and Bulk Average } \\
\text { Panel Temp. Accuracy (K) }\end{array}$ & \pm 0.1 & \pm 0.2 & \pm 0.2 & \pm 0.5 & \pm 2.0 & \pm 3.0 & \pm 3.0 \\
\hline $\begin{array}{l}\text { I/F Point and Bulk Average } \\
\text { Panel Temperature } \\
\text { Resolution (K) }\end{array}$ & 0.03 & 0.03 & 0.033 & 0.1 & 1.0 & 3.0 & 1.0 \\
\hline $\begin{array}{l}\text { Max. Allowed Gradient } \\
\text { within each Panel (K) }\end{array}$ & 2.0 & 2.0 & 2.0 & 5.0 & 7.5 & 10.0 & 10.0 \\
\hline Required Stability (K/hour) & $\pm 0.2 *$ & \pm 0.5 & NA & NA & NA & NA & $\pm 3.0^{* *}$ \\
\hline $\begin{array}{l}\text { Max. Heat Load to/from } \\
\text { interface point or panel }(W)\end{array}$ & 0.5 & 0.5 & 1.0 & 5.0 & 20.0 & 20.0 & 20.0 \\
\hline $\begin{array}{l}\text { Max. Rate of Change of I/F } \\
\text { points and radiant panels } \\
\text { during Transitions (K/hr) }\end{array}$ & NA & NA & 20.0 & 20.0 & 20.0 & 20.0 & 20.0 \\
\hline
\end{tabular}


Figure IV-3 illustrates the Regions 1 and 2 ISIM hardware in the STMS, as it will appear in the SES during the Cryo-Vac tests.

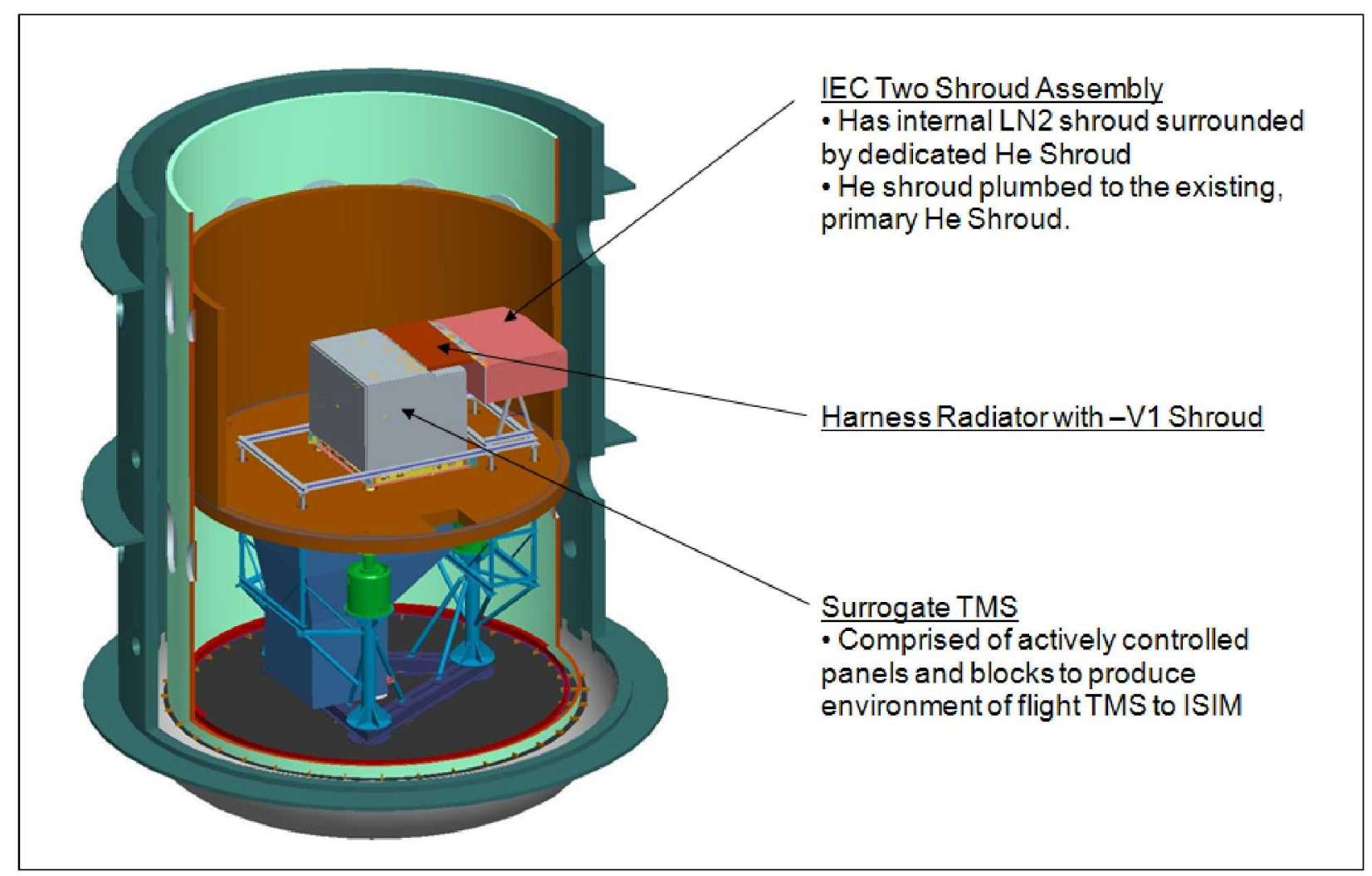

Figure IV-3. Test Configuration of ISIM Regions 1 and 2 inside the GSFC SES facility during Cryo-Vac testing

\section{B. Q-meter Design Considerations}

Model correlation will make use of the flight housekeeping temperature sensors mounted on the ISIM structure, science instruments, harness radiator, etc., plus flight science instrument temperature sensors. It is recognized also that Q-meter design and operation is of critical importance to thermal model validation. A schematic of a typical Q-meter is shown in Figure IV-4. The device as shown is designed to stabilize minor transient temperature fluctuations at the coldplate end (point $\mathrm{C}$ ) by controlled heat

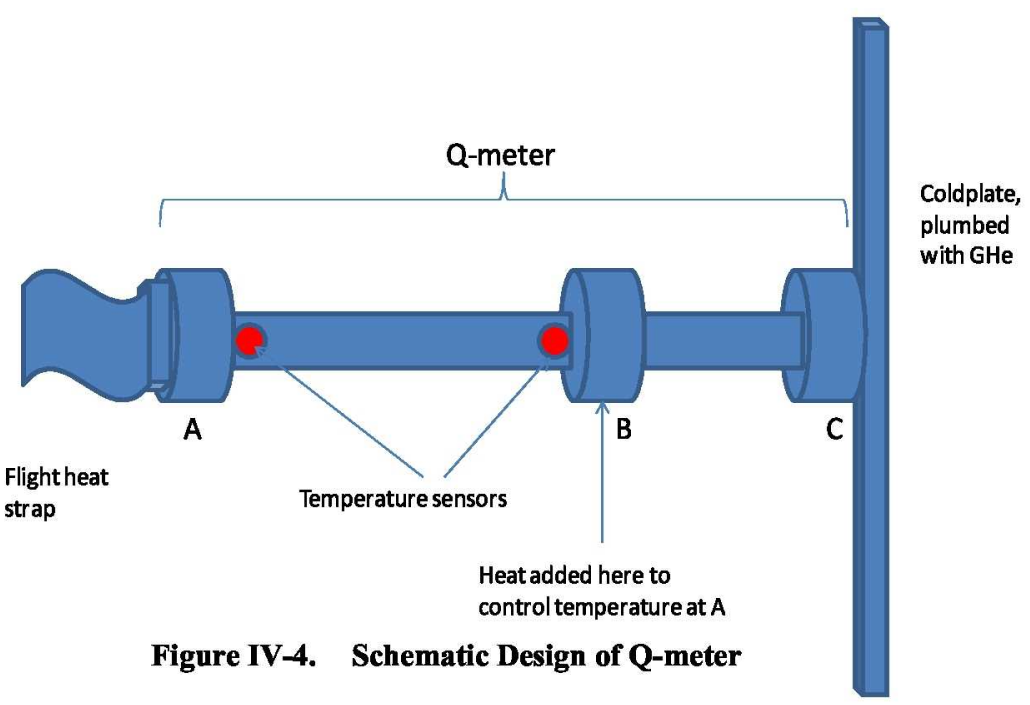
addition at point $B$. Typically, the temperature at point $\mathrm{A}$ is controlled to the predicted radiator temperature that must be maintained in the balance condition. An alternative control approach has been suggested, however, in which temperature controllers are used at both points $A$ and $B$ to maintain a constant temperature difference across the section. Then, heat flow through the device in the test may be inferred by direct comparison of control heat added to that added during device calibration. 
This control approach yields the advantage of being insensitive to Q-meter temperature changes as it is approaching balance (the meter itself will not be absorbing heat). Both approaches will be investigated during calibration.

The Q-meters must be designed to provide adequate resolution of heat flows over the full predicted range under operational (thermal balance) conditions. This results in Q-meter design that provides sufficiently high thermal resistance at cryogenic operating conditions to provide measurable temperature differences across the devices with the predicted steady state heat flows. Temperature sensors across sections A and B in Figure IV-4 must have adequate resolution and repeatability to yield adequate heat flow measurement during the cryogenic balance conditions. In the ISIM Cryo-Vac tests, accelerated transient cooldown will be accomplished by setting all radiant panels and conductive interface points (including the Q-meters) as cold as possible quickly to draw heat out of the instruments and structure. This provides a competing design consideration for the devices: they must exhibit a low enough thermal resistance to heat flow at higher temperatures so that they do not unduly prolong the transient cooldown period. These design tradeoffs are being investigated at this time. Preliminary calculations indicate that the Q-meters, if designed of 6061-T6 aluminum to produce a 1K temperature drop across section A-B during balance conditions, would likely not extend the cooldown period to meet thermal balance stability criteria by more than 1 day. Table IV-4 lists preliminary thermal control and heat flow measurement requirements for the ISIM Qmeters.

Table IV-4. Preliminary Thermal Control Requirements for STMS Q-meters

\begin{tabular}{|l|c|c|c|c|c|c|}
\hline \multicolumn{1}{|c|}{ Parameter } & Notes & $\begin{array}{c}\text { NIRSpec } \\
\text { OA }\end{array}$ & $\begin{array}{c}\text { NIRSpec } \\
\text { FPA/ASIC }\end{array}$ & NIRCam & FGS & MIRI \\
\hline $\begin{array}{l}\text { Predicted min. heat flow at operating conditions, } \\
\text { test/flight (mW) }\end{array}$ & 1 & 61 & 60 & 180 & 79 & 47 \\
\hline $\begin{array}{l}\text { Predicted max. heat flow at operating conditions, } \\
\text { test/flight (mW) }\end{array}$ & 1 & 98 & 93 & 202 & 99 & 61 \\
\hline $\begin{array}{l}\text { Required minimum design heat flow to be measured at } \\
\text { cryo balance (mW) }\end{array}$ & 2 & 15 & 15 & 45 & 20 & 12 \\
\hline $\begin{array}{l}\text { Required maximum design heat flow to be measured at } \\
\text { cryo balance (mW) }\end{array}$ & 2 & 400 & 400 & 800 & 400 & 250 \\
\hline $\begin{array}{l}\text { Required heat flow accuracy at cryo balance (operating } \\
\text { conditions) (mW) }\end{array}$ & 3 & 3 & 3 & 9 & 4 & 2 \\
\hline $\begin{array}{l}\text { Required heat flow resolution at cryo balance (operating } \\
\text { conditions) (mW) }\end{array}$ & 4 & 1.5 & 1.5 & 4.5 & 2 & 1 \\
\hline $\begin{array}{l}\text { Temperature Stability Required at Location "B" during } \\
\text { cryo (balance) (+/- mK/hr) }\end{array}$ & 5 & 7.5 & 20 & 20 & 2 & 20 \\
\hline $\begin{array}{l}\text { Temperature Stability Required at Location "B" during } \\
\text { cryo (non-balance) (+/-mK/hr) }\end{array}$ & 6 & 33 & 33 & 33 & 33 & 33 \\
\hline $\begin{array}{l}\text { Maximum increased time to achieve thermal balance } \\
\text { from ambient cooldown with Q-meters vs. no Q-meter } \\
\text { (days) }\end{array}$ & & 2 & 2 & 2 & 2 & 2 \\
\hline
\end{tabular}

Notes:

1. Taken from ITCS CDR Package, Section 7 (Shaun Thomson), 3-4 March, 2009, p.7-83

2. Minimum flow taken as 1/4 min. predicted from (1). Maximum flow take as 4 times the max predicted from (1). (Min/max q-meter calibration limits)

3. Required Accuracy $=5 \%$ of minimum predicted strap heat flux

4. Required resolution $=$ half of accuracy

5. Required Q-meter thermal boundary stability = half of SI stability criteria at cryo for NIRSpec OA and FGS, $10 \%$ of stability criteria for NIRSpec FPA/ASIC, and $\sim 25 \%$ NIRCam stability criteria. Stability criteria taken from ITCS CDR Package, Section 18 (S. Glazer, D. McGuffey), 3-4 March, 2009, p.18-55

6. Taken from flight requirement of $0.1 \mathrm{~K} / 10000 \mathrm{sec}$ 


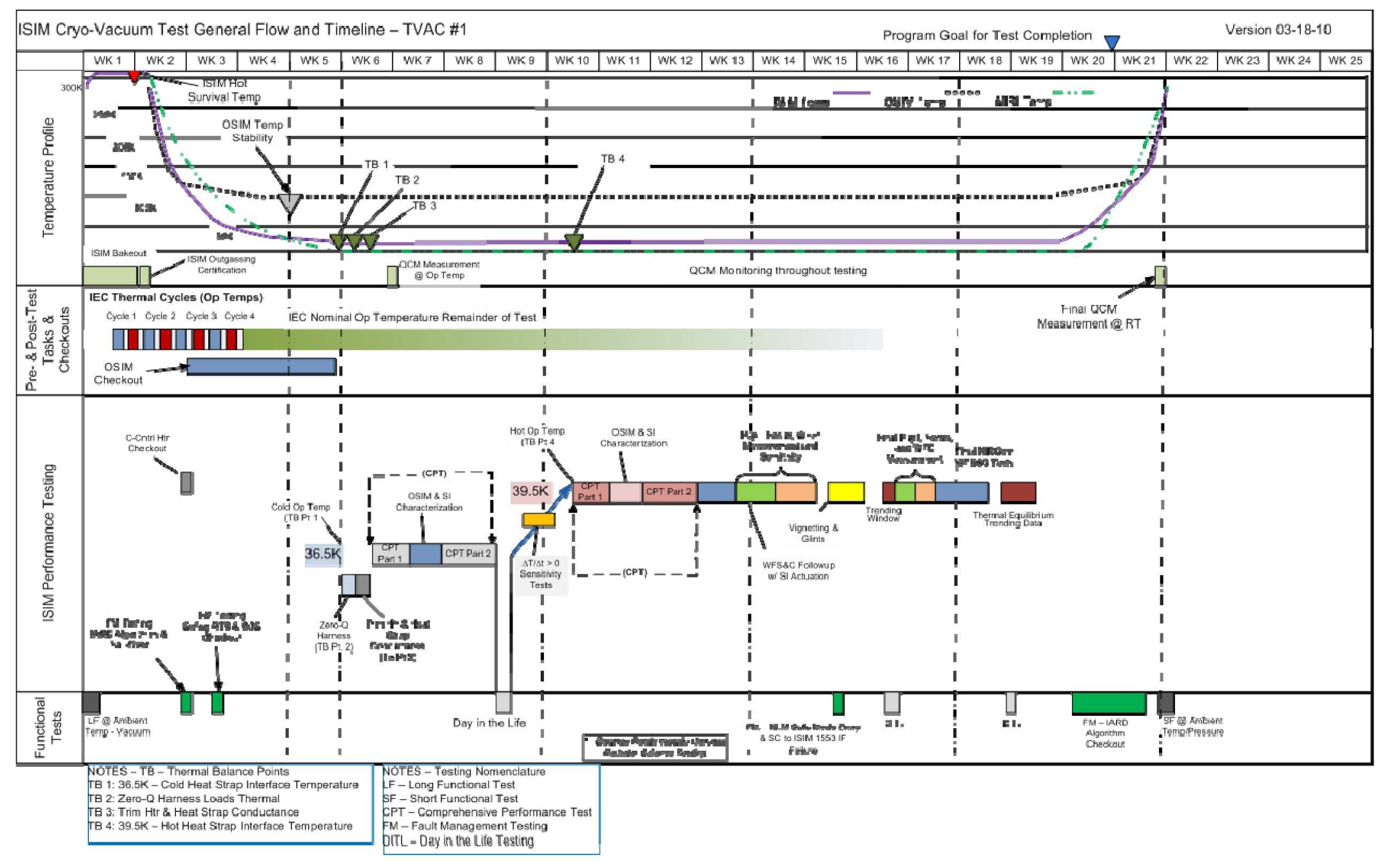

Figure IV-5. ISIM Cryo-Vac Test Profile 


\section{Test Profile}

Intensive ISIM Cryo-Vac Test planning is underway to optimize test measurements while minimizing test times. Currently, both pre- and post-vibration Cryo-Vac tests are expected to last approximately 21 weeks. Figure IV-5 represents the current status of the test profile, and is subject to change as the test planning matures.

Note that the test plans currently call for four balance points;

1. Extreme cold thermal environment with very low instrument power dissipation;

2. Delta balance from (1), with zero-Q of harness heat loads to ISIM;

3. Delta balance from (2), with different trim heater power;

4. Extreme hot thermal environment with high instrument power dissipation.

Balance points 1 and 4 are designed to prove a viable thermal design over the full range of predicted thermal conditions, and to enable model validation over these ranges. Balance point 2 is accomplished by adjusting the temperature of the electrical harnesses as they leave the Instrument Harness Radiator to the same temperature as the ISIM structure attachment points, to provide adiabatic conditions. The difference in heat flows (as measured at the Q-meters, for instance), between balance points 1 and 2 will verify the magnitudes and physical locations of harness heat loads (while great efforts have been expended to predict harness parasitic heat loads, some significant modeling assumptions remain unproven). Balance point 3 is expected to yield information regarding heat strap (between instrument and radiator) end-to-end thermal conductance, plus additional information regarding instrument temperature sensitivity to trim heater power. Requirements for minimum heat strap end-to-end thermal conductance were established because of their impacts to Observatory thermal margins. Maximum heat strap end-to-end thermal conductance requirements were also developed to guarantee that contamination control heater circuits have adequate power to accomplish decontamination in flight. Thermal conductances of individual heat strap segments are being verified by the vendor by test at the component level, but because they must be fastened together in-situ during ISIM integration, joint conductances and their effects on end-to-end conductance cannot be verified until the ISIM CryoVac tests.

\section{Test Risks}

The ISIM Cryo-Vac tests to be performed in 2011 and 2012 at GSFC are extremely complicated, involving complex thermal and optical GSE. The predicted magnitude of the combined dissipated and parasitic heat loads within the ISIM is less than $1.0 \mathrm{~W}$, making quantification of parasitic heat loads, particularly from GSE and other unknown chamber sources, critical. Additionally, model correlation activities associated with other thermal vacuum/thermal balance tests in support of the JWST program have taken months, and have involved assumptions of environmental conditions and thermo-optical properties not readily available at the time of the test.

The possible inability to obtain reliable thermal balance data, and provide flight-like thermal conditions during the test has been recognized as one of the highest risks in the ISIM Risk database. This risk is heightened by the elimination of the ETU Thermal Vacuum test program. Mitigation of the risk is accomplished with the following activities:

1. Allocation of parasitic heat loads to major GSE, such as the OSIM and other optical GSE. Predicted parasitic loads are reviewed, and where possible, parasitics are to be measured in GSE component-level tests;

2. Maintenance of comprehensive lists of all GSE electrical harnesses, predictions of their estimated parasitic heat leaks, and attempts to heat sink the harnesses to specific locations in the test facility;

3. Preliminary characterization of parasitic heat loads in the helium shroud environment using bolometers (often "sneak" heat loads can emerge through vents, MLI closeouts, and small views of the Nitrogen shroud thermal environment);

4. Establishment of the incremental facility and GSE test program in the SES;

5. Constant communications with the instrument design teams throughout the JWST program, to exchange "lessons learned" by all teams during testing;

6. Requirement for key instrumentation and control assets to be fully redundant to minimize chances of test interruptions;

7. Continual re-evaluation and review of test plans by internal and external organizations. 


\section{Conclusion}

Thermal vacuum/thermal balance testing of the James Webb Space Telescope cryogenic flight Integrated Science Instrument Module requires the development of the extremely complex Surrogate Thermal Management System to provide realistic flight-like conditions to the payload, and make accurate heat flow and temperature measurements enabling model validation. Careful model correlation from test data will require fine resolution of expected dissipations and parasitic heat loads from test-induced sources at the milliwatt level. Intensive, multidiscipline integrated planning efforts are underway to optimize the test plans and test profile. Thermal test planning activities will focus on instrumentation accuracy/resolution adequacy for the test conditions, and detailed steady state and transient thermal modeling of the flight ISIM in the SES. While the test is challenging, and possibly represents one of the most complex cryogenic tests ever performed at the GSFC, current indications are that the testing will succeed in flight hardware verification and model validation.

\section{Appendix. Acronyms}

$\begin{array}{llll}\text { ASIC } & \text { Application Specific Integrated Circuits } & \text { JSC } & \text { Johnson Space Center } \\ \text { BIA } & \text { Beam Image Analyzer } & \text { JWST } & \text { James Webb Space Telescope } \\ \text { BSF } & \text { Backplane Support Fixture } & \text { K } & \text { Kelvin } \\ \text { CCE } & \text { Cryocooler Electronics } & \text { KM } & \text { Kinematic Mount } \\ \text { CSA } & \text { Canadian Space Agency } & \text { LN } & \text { Liquid Nitrogen } \\ \text { DM } & \text { Development Model } & \text { MATF } & \text { Master Alignment Test Fixture } \\ \text { EC } & \text { European Consortium } & \text { MIRI } & \text { Mid Infrared Instrument } \\ \text { ESA } & \text { European Space Agency } & \text { MLI } & \text { Multilayer Insulation } \\ \text { ETU } & \text { Engineering Test Unit } & \text { NGAS } & \text { Northrop Grumman Aerospace Systems } \\ \text { FGS } & \text { Fine Guidance Sensor } & \text { NIRCam } & \text { Near Infrared Camera } \\ \text { FM } & \text { Flight Model } & \text { NIRSpec Near Infrared Spectrograph } \\ \text { FPA } & \text { Focal Plane Arrays } & \text { OSIM } & \text { OTE Simulator } \\ \text { GESHA Goddard Equipment Support Hardware } & \text { OTE } & \text { Optical Telescope Element } \\ & \text { Assembly } & \text { PG } & \text { Photogrammetry } \\ \text { GHe } & \text { Gaseous Helium } & \text { PMBSS } & \text { Primary Mirror Backplane Support Structure } \\ \text { GN } & \text { Gaseous Nitrogen } & \text { SES } & \text { Space Environmental Simulator } \\ \text { GSE } & \text { Ground Support Equipment } & \text { SI } & \text { Science Instruments } \\ \text { GSFC } & \text { Goddard Space Flight Center } & \text { STMS } & \text { Surrogate Thermal Management System (for } \\ \text { HR } & \text { Harness Radiator } & & \text { use in test) } \\ \text { HSA } & \text { Heat exchanger stage assembly } & \text { TB } & \text { Thermal Balance } \\ \text { IEC } & \text { ISIM Electronics Compartment } & \text { TMS } & \text { Thermal Management System (flight) } \\ \text { I/F } & \text { Interface } & \text { TV } & \text { Thermal Vacuum } \\ \text { IHR } & \text { ISIM Harness Radiator } & \text { VM } & \text { Verification Model } \\ \text { ISIM } & \text { Integrated Science Instrument Module } & \text { W } & \text { Watt }\end{array}$

\section{Acknowledgments}

S.G. Author would like to thank Mr. D. McGuffey for use of graphics of test plans, Mr. S. Thomson for graphics illustrating ISIM flight and corresponding test configurations, and Mr. W. Bell and Mr. B. Comber for thermal model development and thermal analysis results.

\section{References}

${ }^{1}$ Parrish, K. and Cleveland, P., "Thermal System Verification and Model Validation for NASA's Cryogenic Passively Cooled James Webb Space Telescope (JWST)", ICES Conference, Paper 05ICES-236, 2005

${ }^{2}$ Parrish, K., Glazer, S., Thomson, S., "The Cryogenic Thermal System Design of NASA's James Webb Space Telescope (JWST) Integrated Science Instrument Module (ISIM)", ICES Conference, Paper 05ICES-206, 2005

3Cleveland, P., Glazer, S., Packard, E., "Enhancements to the NASA/Goddard Space Flight Center (GSFC) Space Environment Simulator (SES) Facility to Support Cryogenic Testing of the James Webb Space Telescope (JWST) Integrated Science Instrument Module (ISIM)", ICES Conference 2010 (submitted for publication) 